\title{
Resenha
}

\section{Depression: Causes and treatment ${ }^{1}$}

\author{
Depressão: causas e tratamento
}

Vivian MASCELLA²

In relation to the first edition of the book published in 2009, this second edition, brings significant advances, specifically regarding new terminology and in the classification of disorders, today's terms replace older ones or are included side by side, and new material published since then is coved.

The book is organized into four parts: clinical aspects, experimental aspects, theoretical aspects, and treatment of depression. Part I describes the definition, symptoms, course and prognosis of depression, as well as ratings of mood disorders, depression, psychotic versus nonpsychotic depression, bipolar disorders, involutional depression and schizoaffective disorder. Part II reports biological and psychological studies of depression, including psychoanalytic theory tests. Part III investigates theories of depression, cognition and psychopathology and the development of depression. Part IV describes the main treatments for depression, including the somatic therapies, psychotherapy and the assessment of treatments for depression: random controlled trials.

Part I presents the questions about depression that are present throughout human history. Even though it has been recognized as a clinical syndrome for over two thousand years, an entirely satisfactory explanation of its intriguing and paradoxical features has not been found. There are few psychiatric syndromes in which the clinical definitions are so constant throughout consecutive periods of history. The nature and etiology of depression are subject to different concepts. Some scholars ensure that depression is a psychogenic disorder, while others claim that it is caused by organic factors. A third group believes in two different types of depression: one psychogenic and the other organic.

Schizophrenia and depression constitute the first and second most common diagnoses, respectively, for admissions to psychiatric hospitals in the United States, and the prevalence of depression outside psychiatric hospitals is evaluated as being five times that of schizophrenia. According to the fourth edition of the Diagnostic and Statistical Manual of Mental Disorders (DSM-IV) of the American Psychiatric Association (APA), the likelihood of developing major depressive disorder during life is from $12 \%$ to $15 \%$ for men and from 10\% to $25 \%$ for women. Major depressive disorder is the main reason for disability in strengthened market economies around the world.

In Symptoms of depression systematic studies are described, along with the main complaints and symptoms, including emotional manifestations, such as depressed mood, negative feelings about oneself, reduced satisfaction, loss of emotional bonds, crying spells, loss response to mood, cognitive manifestations, negative self-evaluation, negative expectations, self-

$\boldsymbol{\nabla} \boldsymbol{\nabla} \boldsymbol{\nabla} \boldsymbol{\nabla}$

1 Beck, A.T., \& Alford, B.A. (2011). Depressão causas e tratamento (2a ed.). Porto Alegre: Artmed.

2 Pontifícia Universidade Católica de Campinas, Centro de Ciências da Vida, Programa de Pós-Graduação em Psicologia. Av. John Boyd Dunlop, s/n., Prédio Administrativo, Jd. Ipaussurama, 13060-904, Campinas, SP, Brasil. E-mail: <vivian.mascella@hotmail.com>. 
recrimination, self-criticism, indecisiveness, body image distortion, motivational events, paralysis of the will, desires avoidance, escapism and withdrawal, suicidal desires, feelings of worthlessness, and increased dependency.Somevegetativeand physical manifestations are included, such as loss of appetite, sleep disturbance, loss of libido, fatigability, and retardation.

In the Course and prognosis of depression systematic studies are presented in detail related to the onset of depressive episodes, remission and chronicity. In Classifications of mood disorders, history, validation and reliability are elucidated from the publication of the first DSM-IV of the APA.

In the chapter presenting Non-psychotic depression versus psychotic depression the depressive "psychoneurotic" reaction is described, as well as severe depression with psychotic features and some clinical cases. Regarding bipolar disorders, the history and definition, criteria for diagnosis of bipolar disorder, the relationship between manic and depressive episodes, symptoms of manic phase, behavioral observations of the manic phase, periodicity of manic-depressive behavior, the pre-morbid personality of manicdepressive patients, and questions for further study are explained. Regarding Involutional Depression, it can be concluded that there are serious doubts about the usefulness of this nosological category. A very common belief that involutional depression can be distinguished from other types of psychotic depression based on symptoms was excluded through controlled studies. In the Schizoaffective disorder section, the scarcity of studies in this area is approached, with the lack of data concerning prevalence rates used as an example. This section also mentions the need for further research into the nature, causes and proper classification of this combination of psychosis and mood disturbance.

Part II of the book presents the Experimental aspects of depression and it demonstrates that, despite millions of studies on depression, there is little basic knowledge on the biological substrate of depression. No laboratory discovery related to the diagnosis of a major depressive episode or a manic episode was identified. The results of studies on the neurobiology of suicidal behavior also remain controversial. Positive results regarding exaggerated levels of depression have been consistently related to steroids, sodium retention and changes in patterns of electroencephalogram sleep.

In Theoretical aspects of depression some theories on depression are presented, such as Behavioral Theories,
Cognitive and Evolutionary Theories, Psychoanalytic Theories, Psychotherapy and Psychological Theories, Existentialist Theories, Neurological Theories, Neuropsychological Theories and Biochemical Theories. In the chapter on Cognition and psychopathology the primary triad of depression is described in detail, in order to better clarify the relationships between the diverse phenomena of cognitive, affective, motivational and physical depressions. In The Development of depression the predisposition to depression is exposed, including the formation of concepts, judgments of value and affection, and specific vulnerabilities, with the relationship between stress and depression, and personality organization in depression also revealed.

In part IV Treatments for Depression the main somatic therapies are explained, together with current pharmacotherapy and its main side effects, and resistance to treatment. In Psychotherapy the first therapeutic approaches are illustrated, emphasizing cognitive therapy. The authors analyze the results of randomized controlled trials, with special attention to comparisons between psychotherapy and antidepressant medication. The data show the positive outcome of relapse prevention with cognitive therapy compared to medication treatment, illustrated from a comprehensive review of studies showing that depressed patients treated with psychological interventions had a relapse rate of $30 \%$, in contrast with a relapse rate of $69 \%$ for those treated with pharmacotherapy alone.

The appendix of the book presents Score instructions for negative dreams which indicates a set of unpleasant dreams characterized by a specific thematic content, and representations of a negative dream are exemplified.

The book fulfills the needs of Psychology and Psychiatry professionals, as well as carefully describing the history and evolution of studies on depression. This edition contributes to the development of scientific knowledge of doctors, psychologists and other healthcare professionals. It also evidences the lack of studies on depression, and the divergence that some systematic studies present in relation to one another, which is richly reported.

Received on: 23/8/2011

Approved on: 25/8/2011 